\title{
Norois
}

Environnement, aménagement, société

$190 \mid 2004 / 1$

Varia

\section{Cherbourg, ville-arsenal en crise}

Des décisions politiques aux destinées locales

\section{Fabrice Ripoll}

\section{(2) OpenEdition}

\section{Journals}

Édition électronique

URL : https://journals.openedition.org/norois/77

DOI : $10.4000 /$ norois. 77

ISBN : 978-2-7535-1538-3

ISSN : $1760-8546$

\section{Éditeur}

Presses universitaires de Rennes

\section{Édition imprimée}

Date de publication : 1 janvier 2004

Pagination : 67-84

ISBN : 978-2-86847-939-6

ISSN : 0029-182X

\section{Référence électronique}

Fabrice Ripoll, «Cherbourg, ville-arsenal en crise », Norois [En ligne], 190 | 2004/1, mis en ligne le 03 septembre 2008, consulté le 13 janvier 2022. URL : http://journals.openedition.org/norois/77 ; DOI : https://doi.org/10.4000/norois.77

Ce document a été généré automatiquement le 13 janvier 2022.

(c) Tous droits réservés 


\title{
Cherbourg, ville-arsenal en crise
}

\author{
Des décisions politiques aux destinées locales
}

\section{Fabrice Ripoll}

1 L'histoire de la construction navale militaire, et plus largement de la fabrication des armes, est bien entendu celle d'une activité de production (avec ses bases matérielles, ses techniques, son organisation du travail) dont la spécificité principale est d'être inséparable de l'histoire géopolitique de la société dans laquelle elle s'inscrit.

Cette histoire est aussi celle de la formation de l'État moderne, de sa constitution comme pouvoir central et administration centralisée, ayant en l'armée une institution majeure et permanente grâce à l'exercice du double monopole militaire et fiscal sur l'ensemble des populations sous contrôle. C'est encore celle des constructions culturelles et idéologiques (croyances et identités collectives, doctrines et idéaux politiques) nécessaires à la préparation et surtout à la pratique chroniques de la guerre, au premier rang desquelles on trouve la puissance, la grandeur, l'intérêt supérieur de la France, de la Patrie, de la Nation. C'est enfin et peut-être avant tout celle d'êtres humains, de collectivités, de villes, bien réels, aux destinées prises dans le faisceau de ces multiples processus imbriqués, allant des plus lourdes tendances mondiales aux décisions de quelques-uns, voire d'un seul : roi, empereur, président. Autant d'histoires solidaires, qui semblent aujourd'hui à un tournant.

3 Ce texte ne prétend pas faire le tour de la " question des arsenaux ${ }^{1}$ ». Il sera tout juste possible de rechercher les principales lignes de force et de suivre quelques pistes de réflexion à partir du cas de l'arsenal de Cherbourg. Voici en effet une ville dont la naissance et le développement, la raison d'être fondatrice et la fonction fondamentale, les dynamiques socio-spatiales prennent caractère d'évidences partagées, précisément par le rôle déterminant de «son » arsenal. 


\section{Splendeur et misère des arsenaux de la marine}

4 De la mise en chantier à la mise en « question » des arsenaux de la marine, c'est donc une histoire complexe qu'il faut fouiller, engageant la plupart des plus importants événements et des plus célèbres acteurs de ce qu'on appelle l'histoire de France.

\section{Naissance et industrialisation d'une construction navale militaire étatique}

$5 \quad$ Une longue première période de plus de deux siècles correspond à la lente constitution d'une organisation étatique et centralisée, spécifique et monopoliste, de la production. Les premières pierres en sont posées sous Louis XIV suite aux efforts de Colbert pour construire et entretenir une flotte qui puisse rivaliser avec la Royal Navy britannique. «Un tel effort, mettant en œuvre les plus grands chantiers permanents de l'Ancien Régime, avec Versailles, ne pouvait s'accomplir sans une mobilisation considérable, pour l'époque, de ressources humaines et matérielles, donc sans une intervention déterminante de l'État. » (Bros, 1987 : 87). Cette politique volontariste voire autoritaire se prolongera jusqu'à nos jours, avec une intensité d'engagement financier irrégulière. C'est de ses plans que les arsenaux de la Marine voient le jour au XVII e siècle : Le Havre, Brouage et Brest (1630-1631), Rochefort (1669) ${ }^{2}$, Toulon (1682) - ces trois derniers étant alors les plus actifs. L'arsenal de Lorient n'entre en activité qu'en 1776. Quant à Cherbourg, les petits établissements privés des frères Boulabert y sont réquisitionnés (loués puis acquis) pour créer en 1793 le Service des Constructions Navales qui devient le premier employeur de la ville. Mais surtout, dix ans plus tard, Napoléon Bonaparte y décide la construction d'un beaucoup plus ambitieux "port de guerre de $1^{\text {re }}$ classe " pour doter le littoral de la Manche d'une base navale digne de ce nom. Parallèlement aux créations de toutes pièces, des moyens de production privés continuent pendant encore longtemps d'être sollicités ou achetés pour répondre aux fortes demandes, surtout en période de crises militaires, alors récurrentes. En 1813 par exemple, de nombreux chantiers aux statuts divers sont actifs: Boulogne, Le Havre, Cherbourg, Brest, Lorient, Nantes, Rochefort, Toulon sur l'actuel territoire français mais aussi Gênes, La Spezzia, Livourne, Naples, Venise et Corfou (Coat, 1982).

6 La succession et la conjonction d'événements décisifs durant le $\mathrm{XIX}^{\mathrm{e}}$ siècle et au tournant du siècle suivant font de cette période une ère de mutation. C'est la naissance de la marine moderne avec l'invention puis l'utilisation généralisée et combinée de nouvelles techniques (l'hélice, la vapeur, la cuirasse) et de nouveaux types de navires de guerre dont l'un d'eux deviendra la spécialisation de l'arsenal de Cherbourg pendant tout le $\mathrm{xx}^{\mathrm{e}}$ siècle: le sous-marin. C'est la rationalisation progressive et l'industrialisation tardive de la production (tardive si l'on se réfère à la GrandeBretagne) dont les établissements étatiques obtiennent le quasi-monopole. Fait unique au monde : hors circonstances et contrats exceptionnels, les arsenaux deviennent ainsi pour longtemps les seuls fournisseurs en bâtiments de guerre de leur seul client, la Marine, c'est-à-dire l'État, par ailleurs employeur de leurs personnels (Bros, 1987). C'est en conséquence la réduction et la stabilisation du nombre et des types d'activités des arsenaux comme de leurs personnels. Mais c'est donc surtout la fermeture organisationnelle de la construction navale militaire, système industriel «de loin le plus centralisé » (Collet, 1988 : 34) au sein de ce qui deviendra, dans l'après deuxième 
guerre mondiale, un "méso-système" (Chesnais, Serfati, 1992) de production d'armement à « régulation administrée » (Hébert, 1995).

\section{De la mondialisation des conflits armés à la mondialisation des marchés de l'armement}

7 Après deux conflits mondiaux, l'entrée dans la « Guerre froide » (ère de la dissuasion et de la prolifération nucléaires) est en effet synonyme d'une nouvelle phase d'évolution. Le rôle et l'omniprésence de l'État se renforcent sous l'égide du Général de Gaulle qui entend moderniser et rationaliser l'économie de la France et affirmer son indépendance politique, notamment vis-à-vis de l'OTAN et des États-Unis. La première loi de programmation militaire (1960-1964) de la toute nouvelle Ve République est votée en 1959. La Délégation Ministérielle de l'Armement (DMA) est créée l'année suivante pour unifier le système et fournir aux armées les équipements dont elles ont besoin. La DMA, renommée Délégation Générale pour l'Armement (DGA) en 1977, est ainsi l'unique organe public, industriel et technologique, de la Défense nationale, agent central de la régulation administrée de l'ensemble de la production... jusqu'à sa remise en question au tournant des années 1990 par les gouvernements successifs, de droite comme de gauche, en un nouveau consensus politique d'un tout autre contenu que celui qui avait prévalu jusque-là.

Dès les années 1970, plusieurs décisions révèlent et préparent cette redéfinition de la mise en œuvre comme de la finalité de la production d'armement: les premières coopérations internationales débutent dans les années 1960 (avec la Grande-Bretagne puis la RFA) pour allonger les séries et baisser les coûts unitaires; suivies dans les années 1970 de nouvelles réglementations pour favoriser les exportations (qui allaient déjà bon train depuis un siècle). Et surtout, le GIAT est créé en 1972 pour regrouper les moyens de production de l'armement terrestre mais aussi pour « séparer des activités étatiques celles à caractère industriel de manière à développer dans les arsenaux l'esprit d'innovation, améliorer leur compétitivité et leur dynamisme» (Collet, 1988 : 31). L'objectif est donc clairement posé dès cette époque et c'est cette même logique qui s'imposera ensuite à la Direction des Constructions Navales et à ses arsenaux.

$9 \mathrm{Au}$ nom des bouleversements géopolitiques et économiques internationaux (chute de l'URSS, fin de la bipolarisation, construction européenne et mondialisation) et de la sophistication croissante des systèmes d'armes (donc l'augmentation des coûts de production et surtout de R \& D), la France a vu depuis les années 1990 s'accélérer la mutation de sa Défense nationale sur fond de contraction budgétaire et de baisse du volume d'activités. Dans les écrits officiels et la plupart des articles de presse, c'est contre la « dérive des coûts » et pour leur « maîtrise » que l'industrie d'armement doit se battre aujourd'hui. Il suffit, pour s'en convaincre, de parcourir les sites Internet officiels, les revues comme Cols Bleus, Défense nationale ou Armées d'aujourd'hui sans oublier bien sûr le Livre blanc sur la Défense (1994). Le nouvel objectif: s'adapter aux conditions du marché international de l'armement. Les moyens: modernisation, optimisation, rentabilité, flexibilité, etc. Avec ce changement de «doctrine " (Hébert, 1996) est décidée la restructuration de la production d'armement, c'est-à-dire l'abandon de l'essentiel des principes antérieurs. Les choix politiques, technologiques et budgétaires sont alors systématiquement présentés comme des «ajustements nécessaires », des « contraintes » qui « s'imposent à notre pays » (Richard, 1999 : 34). 
Un processus complexe et multiforme (plus ou moins brutal ou subtil) de désétatisation et plus franchement de privatisation est ainsi partout engagé, avec changement et contournement des statuts faisant de la $\mathrm{DCN}^{3}$, de ses arsenaux et personnels le dernier bastion étatique à attaquer: les activités industrielles ont été regroupées au sein de DCN Ingénierie, «matrice d'une future société nationale ( (Hébert, 1995 : 34). La DCN est mise en concurrence avec l'industrie privée et les autres organisations européenne. Les arsenaux perdent ainsi le monopole de la production (dont ils externalisent une part de plus en plus importante) et sont mis en concurrence les uns avec les autres (en terme de volume et de types d'activités). Ils doivent développer l'exportation de leurs productions militaires, d'où la création en 1991 de DCN-International, « société de droit privé, contrôlée par l'État » (Chesnais, Serfati, 1992 : 93). Ils doivent aussi se diversifier vers des productions civiles. L'embauche des personnels à statut étatique diminue en même temps que l'âge de la préretraite, alors qu'augmente la part relative de personnels en sous-traitance, cette part pouvant précisément varier selon la conjoncture. Les centres de formation initiale des arsenaux sont fermés et reconvertis en centres DGA ouvert à tous et soumis à la concurrence, etc. Le 30 mai 2003, DCN est devenue une entreprise de plein exercice, société de droit privé à capitaux publics. En termes simples et lapidaires, on assiste à « la fin des arsenaux et de l'arsenalisation » (Hébert, 1995 : 114-121).

\section{De l'arsenal à la ville}

11 Cette histoire sans laquelle « les arsenaux demeurent incompréhensibles » (Bros, 1987 : 86) ne doit pourtant pas se réduire pas à cette succession de processus désincarnés. Nul doute que les activités militaro-industrielles aient répondu et répondent encore à des contraintes et enjeux géopolitiques et économiques, organisationnels et financiers, tactiques et stratégiques, de site et de situation, à la fois maritimes, littoraux et terrestres. Mais les arsenaux ne sont pour autant ni de simples points sur une carte d'État-major, ni de simples cases dans un organigramme bureaucratique, ni de simples lignes budgétaires. La réflexion sur le passé comme sur l'avenir des arsenaux ne peut pas faire l'impasse sur les groupes sociaux, les villes, les « sociétés locales » qu'ils ont fait naître ou se développer et qui en sont dépendants, à des degrés divers, au moment des restructurations.

\section{La dimension géographique d'une organisation étatique}

À l'échelle nationale, une division technique et géographique du travail de construction navale s'organise progressivement et tend à se stabiliser à deux niveaux : les arsenaux de Cherbourg, Brest, Lorient et Toulon deviennent complémentaires en se spécialisant, et ceci au sein de ports de guerre "totalement séparés des ports de commerce" préexistant (Bros, 1987 : 87-88). Leurs activités et leur organisation du travail réclament la concentration d'une grande quantité de main-d'œuvre, qui plus est qualifiée, que leur localisation traduit en migrations importantes et leur pérennité en enracinement. Cette exigence à la fois socio-économique et spatio-temporelle (qui n'existe, ne tire sa force et ne peut devenir réalité que par la puissance et l'autorité de l'État) génère ainsi des concentrations ouvrières, isolées les unes des autres mais comptant à l'époque parmi les plus importantes du pays, ainsi que de véritables villes moyennes. Situées le 
long des littoraux et, à part Toulon, à l'ouest de la fameuse diagonale Le Havre/ Marseille, c'est-à-dire à la périphérie de régions rurales elles-mêmes périphériques, celles-ci sont la matérialisation de l'existence et de la puissance militaire et économique de l'État.

Figure $1:$ Cherbourg : la ville et l'arsenal

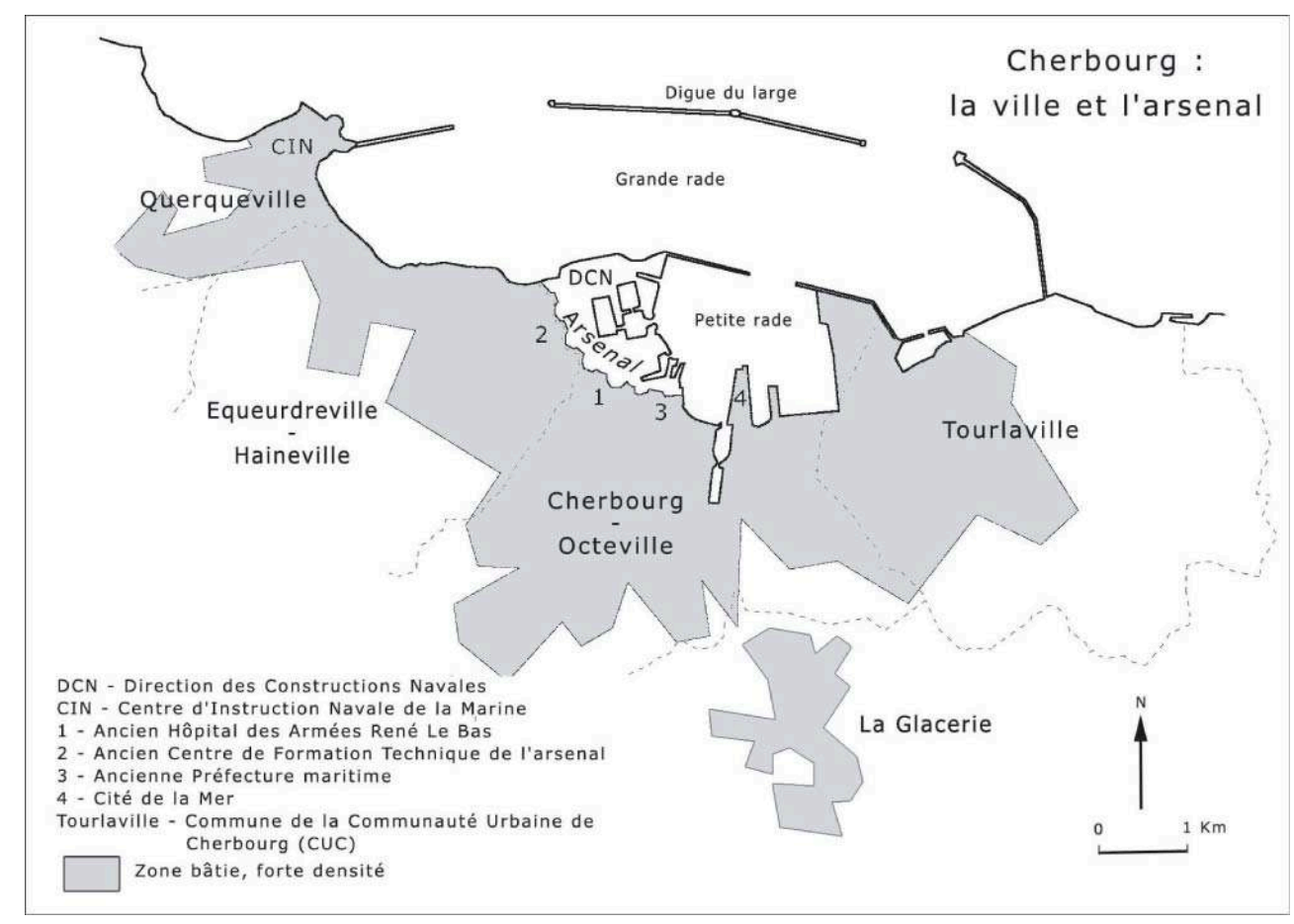

F. Ripoll - 2003

Ces villes, dont Cherbourg constitue une sorte de cas limite, cumulent ainsi l'origine exogène et la position excentrée, l'isolement et l'enclavement relatifs, la faiblesse des relations et l'importance du contraste socio-économique avec leur arrière-pays. C'est pourquoi elles représentent autant d'anomalies pour les rationalisations de l'organisation du territoire qui visent à accorder position dans l'espace géographique et position hiérarchique dans l'armature urbaine, que ce soit en pratique (maillage administratif) ou en théorie (modèles scientifiques comme celui de Christaller). Villes les plus importantes de leur département, elles n'en seront que sous-préfectures (seul Toulon deviendra préfecture... en 1975 !). S'il est vrai que « le port militaire va chercher ce qu'il veut là où il veut " (Vigarié, 1988 : 119), son " espace régional d'implantation " (Fischer, 1994) ne peut qu'être transformé par cette soudaine et durable nouvelle donnée socio-spatiale, impulsant ou absorbant une partie des migrations en provenance du monde rural, déformant la structure urbaine comme les réseaux de transport préexistants, ainsi que les rapports entre les populations et acteurs locaux, entre ceux-ci et les pouvoirs centraux, etc. Pour autant, l'exemple de Cherbourg permet de préciser et de faire ressortir les traits les plus saillants d'un modèle d'analyse articulant principalement, pour rendre compte de ces logiques socio-spatiales, les échelles nationale et locale (urbaine). 


\section{L'arsenal de Cherbourg, moteur des dynamiques locales}

14 effectivement jamais devenues telles qu'aujourd'hui sans les différentes réalisations et activités militaires, portuaires puis industrielles et leurs étroites imbrications. Si l'on remonte assez loin, on ne trouve qu'une place forte gallo-romaine, un village viking fortifié, puis une cité ducale au tournant de l'an mil. Une histoire mouvementée qui n'entraîne pas de développement important de la ville. Au contraire: elle est régulièrement attaquée, change six fois de main et vit plusieurs décennies d'occupation anglaise pendant la «Guerre de Cent ans ». L'essentiel se déroule en fait beaucoup plus tard, entre la fin du XvIII ${ }^{e}$ et le milieu du XIX ${ }^{e}$ siècle.

t abord les grands travaux commencés sous Louis XVI pour édifier contre vents et marées (au prix de dépenses considérables et de nombreuses vies humaines) une digue de 3,7 kilomètres de long, devant constituer une rade protectrice pour de nombreux bâtiments de guerre. Viennent ensuite s'y ajouter ceux de « Port Napoléon ", port militaire couplé à un arsenal de la Marine sans commune mesure avec les premiers chantiers navals cherbourgeois. L'activité des chantiers devient non seulement la plus importante mais la raison d'être d'une ville qui va rapidement se construire à partir et autour de l'arsenal. Pendant ces quelques décennies s'opère en effet à n'en pas douter le tournant de la ville, pour ne pas dire sa naissance en tant que ville, que ce soit en terme d'impulsions démographique et économique décisives, de promotion administrative, de production de l'espace bâti (voire conquis sur la mer), de genèse et de structuration inséparablement économique et spatiale d'une "société locale", relativement indépendante et autonome par rapport à son environnement immédiat (ce qui ne veut pas dire hermétique ou autarcique) mais fortement dépendante voire hétéronome vis-à-vis du pouvoir central (ce qui ne veut pas dire totalement).

Loin de n'être qu'une figure emblématique ou le fruit d'un mythe non fondé, l'arsenal est bel et bien resté pendant plus de 150 ans le pilier socio-économique de la ville, source la plus stable de la plus grande quantité d'emplois directs (jusqu'à 5 à 6000 ) et indirects, de ressources et d'effets induits, avec toute l'importance symbolique que cela comporte. Lieu de rencontre et de confrontation des mondes civil et militaire, de l'État central et de la population locale; lieu d'insertion voire de relative ascension sociale de travailleurs aux origines géographiques et sociales diverses; lieu de construction du groupe socioprofessionnel le plus important de la ville, comprenant de véritables dynasties ouvrières, l'arsenal est aussi depuis un siècle un foyer de militants, de responsables syndicaux et d'élus politiques locaux, impulsant ou soutenant des luttes sociales locales comme nationales, affirmant dans toute la ville une idéologie politique progressiste, certes plutôt modérée et réformiste, mais contrastant avec le conservatisme social de l'environnement rural (Quellien, 1984).

Au début du Xx siècle, André Siegfried dresse ce portrait de la ville : "Cherbourg n'est en réalité autre chose qu'un ancien gros village, démesurément développé autour de l'arsenal, vivant pour et par l'État français: avec sa digue colossale, ses forts redoutables, c'est une création politique. Comme population, il s'agit avant tout d'une ville d'ouvriers. À l'exception d'un milieu restreint de bourgeois, de hauts fonctionnaires, d'officiers de la marine retraités [...], à l'exception encore du moyen et $\mathrm{du}$ petit commerce [...], c'est vraiment la classe ouvrière qui remplit tout. Non seulement elle peuple la ville de ses 43730 habitants, mais elle déborde sur une 
ceinture de grosses communes suburbaines, qui, rurales ou maraîchères à leur extrémité, sont complètement urbaines à leur début, où seul l'octroi fait connaître qu'elles ont commencé [...]. L'ensemble, qui est inséparable, constitue une agglomération de plus de 60000 âmes, dont l'aspect général, avec ses interminables rues de maisons grises et basses, est celui d'une populeuse cité industrielle, surgie de toutes pièces autour d'une immense usine » (Siegfried, 1995 : 413).

Effectivement, des grands travaux à nos jours, les familles ouvrières n'ont jamais été cantonnées dans un espace réservé, dans quelque cité issue d'un paternalisme industriel ou militaire ici inexistant. Alors que dès le début des chantiers, une partie d'entre elles reste ou s'installe dans les villages et hameaux des campagnes alentours (dont la majorité est issue), une autre se regroupe aux abords immédiats de l'établissement, à Cherbourg même et dans des faubourgs qui s'étalent rapidement sur les communes de la plaine littorale (Équeurdreville à l'ouest, aux portes de l'arsenal, puis Tourlaville à l'est). Au fil des décennies, le groupe ouvrier a ensuite investi l'ensemble des communes voire des municipalités de l'agglomération et de sa proximité immédiate, co-produisant toutes les dynamiques urbaines et péri-urbaines cherbourgeoises. En 1996, les 4310 salariés de DCN Cherbourg sont encore présents partout en nombre, avec $13 \%$ d'entre eux résidant dans la ville-centre, $71 \%$ dans l'agglomération et 96 \% à moins de vingt kilomètres (Ripoll, 1996) 4 .

Ainsi, s'il est possible de parler d'une réelle concentration de l'espace ouvrier, elle se saisit à l'échelle de l'agglomération voire du bassin d'emploi, lequel est de fait très ramassé. L'arsenal et la ville tendent ainsi à fusionner en un système d'intégration sociale à double face, inséparablement économique et urbaine, principal vecteur d'identité, de mémoire et de pratiques collectives. Il n'est finalement pas excessif de dire que le groupe des personnels de l'arsenal a formé (par les réseaux familiaux, amicaux, associatifs, syndicaux, politiques, d'entraide et autres relations interpersonnelles hors-travail) une sorte de ciment socio-spatial d'une micro-société peu étendue et contrastée... il y a quelques années encore (Guermond, Muller, 1984 ; Ripoll, 1996). Le fait que le travail et le statut d'ouvrier à l'arsenal aient pu être, au moins dans certaines périodes, valorisés voire considérés comme un privilège en dit d'ailleurs long sur les perspectives offertes à la population par le marché du travail, et partant, sur la structure sociale locale ${ }^{5}$.

Jusqu'aux années 1980, aucune autre activité productive n'a en effet eu l'importance économique et symbolique de l'arsenal. Quelques établissements industriels ont pu avoir une certaine autonomie (par effet de taille et d'agglomération) ou un poids parfois non négligeable, mais toujours secondaires ou provisoires. C'est le cas des Constructions Mécaniques de Normandie (CMN, ex-chantiers aéronautiques Félix Amiot), chantiers privés de construction navale civile et surtout militaire, spécialisés dans les petits bâtiments de surface, qui ont connu leur apogée durant les années 1970 avec plus d'un millier de salariés. Les quelques activités nées de l'expansion industrielle décentralisée des années 1960 (téléphonie, confection), à forte main-d'œuvre féminine et spécialisée, ont quant à elles périclité dès les années 1980, de même que l'éphémère construction de plates-formes de forage en mer (UIE) qui emploiera jusqu'à 1200 personnes en $19766^{6}$. Quant au monde strictement portuaire et civil, après une courte « épopée transatlantique » dans l'entre-deux-guerres et outre les ports de pêche et de plaisance, il est aujourd'hui dominé par une activité transmanche (fret et voyageur) intense mais peu pourvoyeuse d'emplois (Raoulx, 1996). 
21 Il a donc fallu attendre les années 1980 pour que se constitue dans le Cotentin un second pôle socio-économique comparable à l'arsenal, que ce soit par son importance, son activité industrielle, son rôle local d'employeur et de donneur d'ordre et... son rapport à l'État. Il s'est lui aussi constitué en une vague de grands chantiers sur deux sites, dans le nord-ouest de la presqu'île à une vingtaine de kilomètres de Cherbourg: construction d'un "centre nucléaire de production d'électricité " EDF à Flamanville (CNPE); et surtout, extension du centre de retraitement des déchets radioactifs COGEMA implanté dans La Hague dans les années $1960^{7}$. La volonté politique d'indépendance nationale en matière énergétique et le choix du «tout nucléaire » ont eu des répercussions locales énormes (démographiques, économiques, infrastructurelles, paysagères, etc.) qui ne sont d'ailleurs pas exemptes d'ambiguïté, audelà de la non négligeable question écologique et sanitaire ${ }^{8}$. Une fois épuisé l'effet de dopage dû à la manne financière accompagnant la construction des usines, et qui n'a profité que de façon partielle et inégale à la main d'œuvre, aux entreprises et aux collectivités locales ; une fois stabilisé le nombre d'emplois directs ou en sous-traitance nécessaire à leur seul fonctionnement quotidien, l'euphorie laisse place à une réalité plus nuancée. Cette activité controversée ne peut en effet vraisemblablement pas jouer le rôle d'intégrateur socio-spatial que l'arsenal aura pu jouer, ni fonder un nouveau développement économique, tout en entérinant et renforçant la nucléarisation et l'étatisation de la presqu'île, et donc sa dépendance. Aujourd'hui, nombre de PME industrielles et tertiaires locales ne pourraient survivre sans leur activité de soustraitance pour "les trois grands donneurs d'ordre " (DCN, COGEMA et EDF à un moindre degré). Malgré la recherche "d'autres partenaires, parfois très éloignés » (Poisson, 1992 : 34), beaucoup ont déjà disparu.

\section{Sur la notion de ville-arsenal}

22 C'est pour ces multiples raisons que, dans une perspective à la fois descriptive, typologique et explicative, le Cherbourg des deux derniers siècles est ici qualifié de ville-arsenal, ce qui revient à spécifier la ville plus largement comme militaroindustrielle et sous contrôle de l'État. À la lecture de plusieurs auteurs, on peut aisément poser l'hypothèse que ce qualificatif est valable (à des périodes, des degrés et selon des modalités certes divers) pour d'autres villes de France (Brest, Lorient) ou d'ailleurs (comme Plymouth en Grande-Bretagne) ${ }^{9}$.

Cette expression semble par ailleurs préférable à celle de port militaire, qui tend à effacer le fait industriel, pas nécessairement présent dans tous les ports (qu'ils soient militaires ou civils) et sous-entend même à l'inverse une prédominance du fait proprement militaire comme dans le cas d'une ville-garnison (qui serait ici le fait de la Marine). Il est vrai que les grands travaux de la rade de Cherbourg, première pierre du port militaire, sont chronologiquement antérieurs à ceux de l'arsenal. Mais le port militaire n'y a jamais été significatif en ce sens qu'il n'a jamais accueilli durablement de flotte importante et n'a jamais été le moteur de la ville. Il est vrai que l'association arsenal/port militaire est fréquente en France et que Brest et Toulon accueillent des flottes importantes, mais cela n'enlève rien au fait que cette association est contingente. Quant à la présence des militaires dans ces villes, elle est variable et ne se réduit pas non plus à celle de la Marine. Plus pertinente, d'un certain point de vue 
problématique, est l'expression ville d'État. Mais sa généralité ne permet pas de distinguer les villes industrielles des villes administratives.

Il n'existe pas de choix lexical et typologique parfait. L'arsenal n'a jamais épuisé les réalités et dynamiques locales, ni à Cherbourg ni ailleurs, tout en ayant une influence qui dépasse le cadre de la seule agglomération urbaine. Comme toute expression, celle de ville-arsenal est donc réductrice. Cela dit, elle rend mieux compte des principes génétique, structurel et fonctionnel de ce type de formation socio-spatiale ${ }^{10}$ où la logique militaro-industrielle, même littorale, l'emporte très largement sur la logique militaro-portuaire. Quant à qualifier la situation en cours et à venir, situation de mutation rapide entraînée par la désétatisation voire la fin des arsenaux, sur fond de désindustrialisation nationale...

\section{Un avenir sans perspectives}

"Cherbourg se trouve à un tournant de son histoire, en raison du désengagement de l'État qui entraîne la remise en cause de la fonction de ville-arsenal alors que les grands chantiers sont terminés » (Raoulx, 1996 : 98). Les conséquences locales de la succession/ conjonction récente et rapide des restrictions budgétaires, des réorganisations et des réductions du format des armées, des restructurations des industries d'armement, des concentrations géographiques des moyens et des personnels (sur Toulon et Brest où est envoyée, en 1992, la flottille cherbourgeoise), des étalements de programmes, etc. sont de fait synonymes de remise en question globale et surtout inattendue, donc non anticipée, des fondements socio-économiques historiques de la ville. Ce qui ne va pas sans licenciements, non renouvellement de postes, fermetures d'entreprises soustraitantes, etc.

L'arsenal et la ville sont ainsi appelés à se diversifier voire à se reconvertir à plus ou moins court terme. Reconvertir l'arsenal? "Quel que soit le secteur concerné, le textile, les charbonnages ou la sidérurgie, la reconversion industrielle n'est jamais simple. Sans doute est-ce encore plus complexe lorsqu'il s'agit d'industries à finalité militaire » (Penanros, 1995 : 9), a fortiori pour un établissement spécialisé dans les sousmarins à propulsion nucléaire... Reconvertir l'économie locale ? À cette heure, aucune perspective d'alternative, donc d'avenir, viable ne semble pouvoir être dégagée des autres activités économiques existantes, qui subissent la domination de la concurrence (Vigarié, 1988; Raoulx, 1996). Quant au projet de "fast-ships ", ces cargos rapides devant assurer une liaison maritime rapide entre Cherbourg et les États-Unis, il est encore loin de se concrétiser. Comme Brest, Lorient et d'autres, Cherbourg a maintenant son musée maritime, pièce maîtresse des stratégies de promotion urbaine par l'image (ici la «maritimité ») et le développement du tourisme: la «Cité de la mer ", installée depuis 2002 dans l'ancienne gare maritime, a coûté 150 millions de francs (dont 25 pour la reconversion du Redoutable, premier sous-marin nucléaire français ouvert à la visite). Mais peut-elle vraiment être le nouveau moteur économique ? Et que peut-on attendre des stratégies de développement de la formation supérieure (IUT, DEUG universitaires, formations d'ingénieur) ? Si, « à Brest comme à Cherbourg, le développement de la fonction universitaire et notamment de formations spécialisées [...] constitue un élément majeur des politiques de promotion et de changement d'image des villes » en même temps qu'il est considéré comme « un enjeu 
majeur économique, social, politique du développement local » (Séchet, 1992 : 91, 93), il semble en fait loin de pouvoir répondre à ces attentes ${ }^{11}$.

Une série de décalages se conjuguent ici, produits directs des logiques à l'œuvre dans la mise en place de la construction navale militaire en France telle que décrite plus haut: une ville-arsenal est greffée au sein d'une région rurale périphérique avec laquelle les rapports ne se développeront pas suffisamment pour permettre une activité économique autonome ou endogène. Décalage donc entre l'importance économique relative que représente l'activité de l'arsenal à l'échelle nationale (pour l'État) et son importance à l'échelle de la formation socio-spatiale locale qu'il a fait naître. Décalage entre la relative facilité de diminution et de réallocation des ressources budgétaires étatiques et la difficulté de gestion locale des pertes sèches ainsi entraînées (pour l'arsenal, les entreprises sous-traitantes, les collectivités territoriales, etc.) et des reconversions ainsi nécessaires (du bâti, des outils et des hommes). Il est donc autant question d'échelle socio-spatiale que d'échelle de temps, de rythmes du changement. Et puis décalage entre les moyens matériels, économiques, sociaux et culturels nécessaires à un développement local "alternatif » et endogène, et les moyens réellement disponibles.

Ainsi, considérer comme c'est souvent le cas que l'avenir de Cherbourg est entre les mains de ses habitants, que ceux-ci ne doivent plus "tout attendre de l'État ", qu'ils doivent au contraire «sortir de cette apathie endémique se traduisant par un manque d'esprit d'entreprise et d'innovation pour conquérir de nouveaux marchés » (Tollemer, 1997 : 53) et « résoudre le brûlant problème de la reconversion et de l'intégration des salariés et des chômeurs non qualifiés " (Lesachey, 1992: 40) revient à prendre pour argent comptant les discours dominants. C'est ignorer le poids des inégalités entre les villes ou régions en concurrence (ou plutôt entre populations et groupes sociaux) : inégalités d'équipements, de ressources, de distance aux centres ou d'accessibilité ${ }^{12}$, d'image, etc., donc des chances de subsistance si ce n'est de "développement " (Vigarié, 1988). C'est donc oublier le processus quasi-circulaire du jeu de l'accumulation/concentration qui favorise les joueurs déjà favorisés. C'est faire preuve d'un volontarisme optimiste mais naïf ("quand on veut, on peut»), qui ignore ou minore la faiblesse des potentialités et des marges de manœuvre réelles. C'est appliquer aux formations socio-spatiales en crise le discours de responsabilisation des victimes ("on n'a que ce que l'on mérite ») répandu dans l'interprétation de nombre de situations difficiles, toutes socialement construites : échec scolaire, chômage, pauvreté, discrimination des femmes, des personnes d'origine étrangère, etc. (et qui n'est que la nécessaire figure complémentaire et opposée du discours méritocratique affirmant que «l'on mérite ce que l'on a »).

\section{Une fatalité historique?}

L'importance de l'arsenal a l'avantage de permettre une lecture géo-historique rapide de Cherbourg qui ne réduise - donc ne trahisse - pas trop une réalité toujours plus complexe. En revanche cette lecture, comme celle de la construction navale militaire française proposée ici, ont les inconvénients de toute reconstruction a posteriori de l'histoire qui sélectionne une série d'événements (parmi d'autres) et les présente sous forme d'un enchaînement chronologique (avec un début, des étapes et une fin). Un premier écueil est celui du finalisme qui prendrait le présent non seulement pour 
l'aboutissement mais pour la finalité d'un projet préexistant, voire pour un destin (ambiguïté du terme de vocation trop souvent employé). De plus, au-delà ou en deçà de toute intentionnalité, par la seule opération formelle de mise en ordre qui la constitue comme récit, la chronique tend à se voir conférer une cohérence logique intrinsèque qu'elle n'a ni nécessairement ni absolument. Enfin, en ne posant pas la question des devenirs potentiels non réalisés, la vision rétrospective ferme un champ des possibles souvent plus ouvert qu'elle laisse à penser, faisant ainsi planer un voile de déterminisme non questionné. D'autres types de lecture doivent donc être menés pour défataliser l'histoire effective, faite et à faire, sans abandonner pour autant le projet explicatif ni minimiser la force des processus sociaux, le poids des héritages, l'irréductibilité des contraintes de l'action, et finalement, l'existence d'incontestables " effets de destin ».

\section{Quelques ratés de l'histoire}

Un résumé rapide de l'histoire de l'arsenal de Cherbourg déduirait sa naissance de la situation géostratégique de la presqu'île. Que penser alors du fait que, malgré l'ancienneté des conflits franco-anglais, donc malgré une situation géostratégique durablement favorable, la décision de construire la digue, le port militaire et l'arsenal a été prise si tardivement? Dans ce type de configuration, une base navale avancée dans le Cotentin est en effet un atout énorme pour accueillir et protéger une flotte importante, contrôler ainsi la mer de la Manche et son trafic, s'opposer aux ports de l'ennemi anglais et pourquoi pas, lui porter le coup décisif. Son absence est à l'inverse un terrible handicap. Cette situation est d'ailleurs repérée par Vauban qui propose un plan de travaux à Louis XIV en 1686. Elle est tout simplement prouvée par les faits six ans plus tard, lors de la Bataille de La Hougue, qui de victoire se transforme en défaite faute d'une rade protectrice dans la région pour les navires français dispersés et affaiblis. S'il est vrai que six ans n'auraient de toute façon pas été suffisants pour achever les travaux à temps, soulignons qu'ils avaient entre temps été interrompus et rasés. Et malgré cet événement, il faut encore attendre 1783 - donc un siècle de plus ! pour que la première impulsion importante soit donnée par Colbert qui convainc Louis XVI de lancer la construction de l'actuelle grande digue du large. Pourtant, de la Révolution au début d'une guerre contre toute l'Europe (dont l'Angleterre et même la Hollande, les deux principales puissances maritimes), les «crédits» vont baisser jusqu'à l'arrêt total des travaux en $1791 . .$.

31 Cette série de "ratés» de l'histoire cherbourgeoise invite à relativiser le rôle de la situation géostratégique dans le développement de la construction navale militaire et donc de la ville elle-même. Un argument sérieux pour rendre raison de ces absences ou retards de décisions serait de dire que la prise de conscience de cet avantage potentiel ne suffit pas à contrebalancer l'inconvénient tout aussi objectif des efforts financiers que les travaux réclament. De fait, l'argument budgétaire arrive en tête des justifications des baisses d'activité récurrentes des établissements militaires. Pourtant, ce n'est visiblement pas le point de vue de Napoléon Bonaparte qui non seulement décrète leur reprise en 1803 mais y ajoute, comme on l'a vu, la construction d'un " arsenal complet avec des chantiers capables de construire tous types de navires" (Taillemite, 1991:6), et ceci malgré de nombreux et importants obstacles financiers, matériels et géographiques. S'ajoutant aux constructions navales à l'ancien arsenal et au chantier de Chantereyne, les grands travaux de surélévation de la digue du large et 
de construction du nouvel arsenal doivent ainsi être menés de front alors que la guerre a non seulement détruit mais ruiné la Marine française, que les ravitaillements (principalement en bois et nourriture) sont particulièrement difficiles, que les chantiers manquent d'ouvriers, principalement d'encadrement (les maîtres), que la ville manque de logements (dont les prix montent en flèche), etc. (Coat, 1980 ; Épin, 1990 ; Lepelley, 1990). De plus, malgré l'ampleur du projet local, le site de Cherbourg n'est pas le seul à "bénéficier » de cette politique et n'est même pas le plus «favorisé » (Anvers devient un chantier plus important encore).

Notons que les efforts fournis - dont les familles ouvrières sont les premières à payer le prix (Épin, 1990) - arrivent trop tard pour empêcher la défaite : l'Empereur abdique en 1814 devant l'invasion prussienne. L'inactivité des chantiers cherbourgeois entre 1791 et 1803 représentent-elle « 12 ans de perdus et qui pèseront lourd [...], 12 ans qui ne se rattraperont jamais » (Lepelley, 1990) ? Rappelons qu'un siècle entier sépare la prise de conscience de Vauban (1686) du premier engagement massif de l'État (1783). Pour Cherbourg, il semble raisonnable de penser que les travaux effectués, aussi tardifs et vains qu'ils peuvent avoir été du point de vue des ambitions napoléoniennes, ont pesé lourd sur sa fonction à venir de ville-arsenal. Et pourtant, ceci est aussi à relativiser : que dire du fait que les sommes considérables déjà engagées ainsi que l'état bien avancé des travaux en ce début de XIX ${ }^{e}$ siècle vont très inégalement convaincre les gouvernants successifs d'apporter une pierre à leur poursuite, et ceci dans un monde loin d'être apaisé? C'est sous le Second Empire, plusieurs décennies après le premier, que l'essentiel est achevé : la digue du large en 1853 et le dernier bassin de l'arsenal en 1858, inauguré en présence, non seulement de l'Empereur, mais aussi, magnifique ironie de cette histoire... de l'ennemi qu'on aurait cru héréditaire et contre lequel l'arsenal et le port militaire furent conçus : la Reine Victoria d'Angleterre! Autrement dit, l'outil est opérationnel au moment où sa fonction originelle n'est plus d'actualité et où sa position stratégique devient mineure.

Le $\mathrm{xx}^{\mathrm{e}}$ siècle et ses conflits (effectifs et potentiels) révèlent ensuite une importance croissante des données technologiques. En ce qui concerne la division du travail entre les établissements et donc la pérennité de l'arsenal de Cherbourg, l'affirmation progressive d'une spécialisation dans la technologie sous-marine sera particulièrement mise en valeur par le rôle croissant de celle-ci durant le siècle, et surtout par la politique du Général de Gaulle en matière de dissuasion nucléaire. Or, cette activité, pratiquée au début du siècle dans deux autres arsenaux (Toulon et Rochefort) aurait très bien pu être pérennisée dans un autre chantier. Rien ne prédestinait ni n'imposait le choix de l'arsenal de Cherbourg dont l'activité sera d'ailleurs remise en cause, notamment en 1921-1922 (Lavalley, Lemonnier, 1999).

Plus récemment, la refonte de l'ensemble du secteur production de DCN Cherbourg (nom officiel de l'arsenal) opérée de 1982 à $1992{ }^{13}$ pour construire une nouvelle génération de sous-marins nucléaires lanceurs d'engins (les SNLE-NG type Le Triomphant), est facilement interprétable comme une confirmation de ce rôle majeur conféré à la dissuasion par le biais de la force océanique stratégique (FOST). Pourtant, dès 1991-1992 (c'est-à-dire à peine ces travaux terminés), les décisions sont prises d'étaler le plan de charge, de retarder et même d'annuler certaines des constructions sous-marines prévues ${ }^{14}$. De plus, ces décisions, qui entraînent une augmentation du coût unitaire des matériels, sont prises au nom d'une réduction et d'une gestion toujours plus rationnelles des dépenses. 


\section{Le secteur de la défense nationale comme champ social} budgétaires, technologiques), ni même leur combinaison, ne sont suffisants pour rendre compte de l'histoire de l'arsenal de Cherbourg. Autrement dit, considérer comme un facteur causal ce qui est plutôt un contexte qui situe l'action (des stratèges, décideurs, exécutants) relève d'une vision trop mécaniste. De même, présupposer que les contraintes objectives, les atouts et handicaps, sont tous et toujours perçus et pris en compte ne fait que déplacer le déterminisme en le transférant de la pure nécessité des facteurs à la parfaite rationalité des acteurs. Il existe toujours le choix entre des objectifs différents, des stratégies et des moyens alternatifs plus ou moins risqués ${ }^{15}$. Or, les dirigeants successifs n'ont pas nécessairement les mêmes ambitions, ni la même clairvoyance, ni la même propension à prendre des risques. Bref, tout processus de décision passe par une évaluation de la situation nécessairement partielle et partiale, inséparablement cognitive et éthique, engageant un système de pensée et de valeurs qui oriente et justifie l'action (Boltanski, Thévenot, 1991) ${ }^{16}$. En décidant les grands travaux de Cherbourg, Louis XVI et Napoléon Bonaparte ont-ils fait la seule chose à faire ou ont-ils fait ce qu'ils ont voulu ? Ont-ils fait preuve de plus de rationalité ou ontils eu la folie des grandeurs ${ }^{17}$ ? Que penser des ambitions du Général de Gaulle ou des décisions actuelles? Seules quelques pistes peuvent ici être lancées mais il faut convenir que ces décisions ne sont ni strictement nécessaires, ni purement rationnelles, ni même absolument raisonnables. Pour les comprendre, il faut considérer qu'elles ont une origine, une pertinence et des conditions avant tout sociales.

En premier lieu, l'État, puisque c'est lui qui occupe le devant de la scène militaroindustrielle, ne peut pas être considéré comme une simple courroie de transmission entre dynamiques supra et infra-nationales. Son rôle est celui d'une "puissance" relativement autonome en ce sens que les décisions au moins partiellement arbitraires des gouvernants ne sont pas réductibles à de simples retranscriptions inévitables des nécessités extérieures et pourraient ne pas être prises au profit d'autres décisions, elles-mêmes partiellement arbitraires. À l'inverse, le pays n'est jamais soumis au pur arbitraire de quelques hommes usant et abusant d'un appareil omnipotent, pouvant décider et faire n'importe quoi en toute liberté. Le pouvoir d'État ne s'exerce que dans les limites de sa capacité à contraindre et convaincre les membres de la société et les autres États. Son action en propre est donc d'autant plus déterminante que les gouvernants disposent à la fois d'une plus grande autonomie de décision et de plus grands moyens d'action leur permettant de ne pas subir les volontés des autres protagonistes ni les tendances historiques, mais, au contraire, de prendre des initiatives et de peser sur le cours des événements. Aussi importante que soit la capacité à s'informer et à anticiper, l'expression " gouverner, c'est prévoir » est de ce point de vue non seulement réductrice mais trompeuse. La prise en compte du rôle et $\mathrm{du}$ fonctionnement de l'État dans l'analyse est finalement d'autant plus indispensable qu'il a ce pouvoir à la fois de faire advenir ce qu'il décide et de décider ce qui doit advenir, autrement dit, qu'il a une position dominante dans les rapports de force et de légitimité dans lesquels il est engagé (et inversement, elle l'est d'autant moins qu'il perd en autonomie, en moyens, en légitimité, etc.).

Bien qu'il existe en France de nombreux facteurs de centralisation administrative, de concentration des pouvoirs politiques et d'unification idéologique de ses agents, l'État 
n'est pas une entité monolithique fermée sur elle-même et sans contradictions internes. Les gouvernants sont dépendants des individus et institutions qui conditionnent la prise et l'application de chaque décision et permettent l'exercice du pouvoir, et plus largement de tous ceux qui ont partie liée avec l'État même s'ils n'en font pas partie (ce que le couple État/société civile ne permet pas d'appréhender). Les mécaniques du pouvoir ont ainsi d'autant plus de jeu que l'État perd en autonomie, que son personnel politico-administratif est hétérogène (Birnbaum, 1980: 11), que s'allongent « les chaînes de délégation de l'autorité et de la responsabilité » et « que le champ du pouvoir se différencie » (Bourdieu, 1997 : 66, 67).

Pour rendre raison de l'histoire de Cherbourg en tant que ville-arsenal, il faudrait donc pouvoir connaitre l'évolution du poids respectif et des rapports mutuels entre tous les agents impliqués dans ces jeux de pouvoir où se jouent l'existence et le fonctionnement de l'arsenal de Cherbourg, de l'industrie d'armement et de l'ensemble de la Défense nationale. Ceci revient à étudier ce secteur d'activité comme un champ social tel que défini par Pierre Bourdieu : univers social relativement autonome, à la fois système de relations sociales constitué autour d'un enjeu commun et spécifique, et structure de positions sociales hiérarchisées en fonction des propriétés et ressources inégalement distribuées qui y sont valorisées et efficaces. Ainsi, les rapports entre les différentes composantes du secteur de production et d'utilisation de l'armement sont des rapports de concurrence autant que de complémentarité, des rapports de force autant que de fonctionnalité, bref des rapports sociaux (dont certains sont des rapports interpersonnels) et non seulement techniques. Chaque décision concernant la Défense nationale s'inscrit dans ces rapports où chacun défend à la fois un point de vue (rationnel et éthique), des intérêts (économiques et symboliques) et même une existence (personnelle ou catégorielle), c'est-à-dire une fonction technique et une position sociale. Il faut donc à la fois saisir la place de chacun dans le champ de la Défense nationale (ou champ militaire) et la place de celui-ci dans le champ du pouvoir d'État.

Il n'est pas possible ici de mettre en œuvre la méthode sociologique associée à cette théorie. Toutefois, en tant que regard, elle offre quelques éclairages intéressants. On peut se faire une première idée de la composition du champ de la Défense nationale à partir de l'organigramme officiel. En haut de l'échelle, on trouve le Président de la République, des membres du gouvernement et des cabinets ministériels (en premier lieu le Premier Ministre et les ministres de la Défense et des Finances), des parlementaires, des hauts fonctionnaires civils, les officiers d'états-majors, les dirigeants de la DGA, des propriétaires et dirigeants d'entreprises, et même des conseillers, experts et chercheurs engagés à titres divers. En bas, les militaires du rang, les petits fonctionnaires et les ouvriers des arsenaux, ainsi que les salariés d'exécution des entreprises privées concernées. Les ingénieurs de l'armement (IA, issus majoritairement de Polytechnique occupent quant à eux des positions à différents niveaux décisionnels intermédiaires et, en tant que corps de l'État, une place globalement centrale au sein du dispositif industriel dont ils assurent une grande part de la « cohérence ${ }^{18}$ » (Chesnais, Serfati, $1992: 69-73$ ).

D'autres agents, absents de cet organigramme, doivent encore être intégrés. Tout d'abord, les autres membres du gouvernement, l'opposition, et l'ensemble des organisations partisanes intervenant dans les orientation politiques, les questions budgétaires ou autres. Mais aussi les organisations syndicales de travailleurs, à 
commencer par celles des arsenaux : créées pour résister aux pratiques coercitives de l'État (enrôlements, levées, déplacements, immobilisations, discipline, etc.) et améliorer leurs conditions de travail et d'existence, elles ont un rôle incontestable quant au « compromis social» nécessaire à la production (Hébert, 1995). Si plusieurs organisations y sont en concurrence (en terme de doctrine et de stratégie), elles se sont aussi fédérées entre elles à l'échelle nationale et solidarisées avec celles d'autres travailleurs et secteurs d'activité (constituant des unions et confédérations à différentes échelles). Ensuite, l'organisation de la construction navale militaire ayant produit des formations socio-spatiales de type ville-arsenal, l'ensemble de leurs membres se trouvent, de fait, fortement concernés par la Défense nationale. Cette interdépendance est d'ailleurs illustrée par les prises de position des cadres syndicaux et élus locaux sur ces sujets (qui s'étonnerait que des élus cherbourgeois défendent la Marine et le choix, forcément rationnel, de l'arme sous-marine?), ainsi que par l'ampleur des manifestations lors des conflits importants (comme en 1992) ${ }^{19}$.

Dans tous ces cas, les interdépendances sociales, économiques, politiques et symboliques jouent un rôle important dans les rapports sociaux au sein du champ militaire. Elles se traduisent par des solidarités ou des tensions au sein des champs syndical et politique (entre le pragmatisme et le doctrinal, entre le local et le national ou, dans les « étatsmajors » parisiens, entre le sectoriel et le général, etc.). Enfin, et plus largement, et parce que la question du militaire engage l'ensemble de la société, il ne faudrait pas négliger le rôle des confrontations politiques (électorales ou non) et idéologiques, donc celui des associations et mobilisations collectives « citoyennes » investissant «l'espace public» (pacifistes, anciens combattants, étudiants réclamant «des sous pour l'éducation, par pour les canons ", etc.) et, plus quotidiennement, celui des journalistes et autres personnalités médiatisées, faisant autorité scientifique et/ou morale, et prenant publiquement la parole sur ces questions.

\section{« Lois du marché » vs « raison d'État »?}

Pour Chesnais et Serfati, «c'est seulement en tenant compte de la place de l'armée dans la société française depuis la monarchie absolue jusqu'au $\mathrm{xx}^{\mathrm{e}}$ siècle et surtout du rôle central qu'elle a joué dans la naissance et les crises initiales de la $\mathrm{V}^{\mathrm{e}}$ République qu'on peut comprendre le caractère extraordinairement timide des tentatives faites par certains gouvernements depuis 1974 pour brider les grands programmes d'armement (dont ils appréciaient le coût financier très élevé, l'intérêt stratégique problématique et peut-être la faiblesse des retombées technologiques) de même que l'abandon presque immédiat des décisions prises au début du septennat de Valéry Giscard d'Estaing comme du premier septennat de François Mitterrand de réduire les exportations d'armes » (Chesnais, Serfati, 1992: 12, souligné dans le texte). Que penser dans ces conditions de l'actuelle mutation du système de production d'armement, sachant qu'elle est présentée, comme naguère la création de la DGA, comme une modernisation souhaitable et nécessaire, c'est-à-dire justifiée par la rationalité des dirigeants d'un État devant s'adapter à l'évolution inéluctable de la situation géopolitique et économique mondiale? Difficile de répondre sans mener une étude approfondie des transformations du champ militaire, de sa structure interne, de sa place et de son autonomie au sein du champ du pouvoir. On peut néanmoins poser quelques questions ou hypothèses quant aux lignes de force de ces transformations. 
43 Les institutions militaires n'y ont-elles pas perdu en légitimité et en autonomie au profit des institutions économiques (commerciales et financières)? Les acteurs et logiques du secteur privé ne sont-ils pas de plus en plus pris en modèle au sein même du secteur public? Pour Jean-Paul Hébert, le « système est frappé de plein fouet par la contestation de la validité du rôle économique de l'État. Cette délégitimation du secteur public est sensible y compris parmi les travailleurs (affaiblissements des solidarités, perceptions de "privilèges »), l'affaiblissement des organisations syndicales ayant transformé les conditions d'élaboration du compromis salarial » (Hébert, 1995 : 10). La finalité de la production d'armes n'est plus d'assurer au mieux la protection de la Nation, quel que soit le prix des moyens nécessaires pour réaliser cet objectif (logique $d u$ «financement d'efficience»). Un glissement s'est opéré: la demande d'une meilleure gestion de crédits en diminution s'est transformée en une demande de rentabilité. Une logique de "part de marché » prend le pas sur l'ancienne logique "politique» (Hébert, 1995 : 123). Il ne s'agit plus de baisser le coût inévitable d'une activité étatique considérée à tord ou à raison comme socialement nécessaire, mais d'assurer des profits grâce à une activité commerciale considérée comme économiquement rentable, pour peu que chacun fasse des « efforts » pour " comprimer les coûts et pour optimiser l'emploi des matériels » (Richard, 1999: 4), " améliorer la compétitivité de nos entreprises» et "mieux les engager dans la compétition internationale ${ }^{20}$ ». Ainsi, la DCN a mené une "vigoureuse offensive commerciale » qui, après l'accord avec le Pakistan pour trois sous-marins type Agosta, a permis la cosignature (avec le chantier espagnol Empresa Nacional Bazan) d'un contrat pour la fourniture à la marine chilienne de deux sous-marins de type Scorpène, et ceci «au terme de deux années de très vive compétition internationale, dans laquelle étaient représentés les principaux fournisseurs de sous-marins dans le monde ${ }^{21}$ ». La course aux armements est plus que jamais une course aux ventes d'armements.

Autrement dit, tout semble indiquer que le secteur militaire est en train de perdre l'autonomie relative qu'il avait en tant que champ étatique, en étant soumis, non pas aux impératifs éthico-politiques d'un désarmement contrôlé, mais aux règles du jeu pragmatiques d'un champ économique en cours de mondialisation. La raison d'État, qui régnait d'autant plus dans les affaires de la guerre que celles-ci sont au fondement de l'État moderne ${ }^{22}$, semble laisser progressivement sa place aux lois du Marché. L'État lui-même (et sa forme nationale territorialisée) est souvent considéré comme non seulement impuissant mais en décomposition face aux bouleversements liés à la mondialisation économique et financière. Ce mouvement est-il inéluctable ou accompagné voire produit par les agents de l'État (paradoxe qui resterait à expliquer)? Outre la pression des grands groupes de sociétés multinationales, un élément d'explication de ce paradoxe résiderait, selon Frédéric Lebaron, dans la "montée en puissance " de la "croyance économique ", dans sa version «libérale» et "monétariste ", via celle des agents qui la portent et la légitiment au sein du champ du pouvoir : économistes chercheurs (surtout d'institutions comme l'INSEE), enseignants (surtout des grandes écoles), experts, syndicalistes, dirigeants politiques, hauts fonctionnaires, managers, journalistes, etc. (Lebaron, $2000: 210-211)^{23}$. Selon cette croyance, la «science économique» permet seule d'appréhender les «réalités économiques ", connaissance rationnelle qui autorise et commande le "réalisme » des " politiques économiques », seul garant de l'intérêt général. «Cette nécessité s'exprime d'une manière extrêmement cohérente dans la doctrine produite par les principales organisations internationales, qui constitue la forme mondialement dominante de la 
croyance économique : Fonds monétaire international, Banque mondiale, OMC, OCDE et, à une échelle régionale, la Commission européenne. Tout gouvernement est soumis à de fortes pressions à la conformité, puisqu'il ne peut durablement s'endetter pour financer ses déficits publics, et doit ajuster son économie à l'impératif d'équilibre en la restructurant autour des secteurs exportateurs «efficaces », en limitant la dépense publique (ce qui passe souvent par la privatisation et l'ouverture aux capitaux internationaux), et bien sûr en combattant systématiquement les pressions inflationnistes" (p. 210-211). On reconnaît ici les principes présidant aux restructurations du système de production d'armement: celui-ci ne saurait faire exception à la (nouvelle) règle ${ }^{24}$.

Quelles qu'en soient les raisons, à coup sûr multiples, ces restructurations ne sont pour l'heure que facteurs de destructuration des formations socio-spatiales locales à partir desquelles ce système productif national s'était constitué. Remise en question d'une vocation? Mais peut-on seulement parler de vocation ${ }^{25}$ ? Après la matérialisation en plusieurs villes-arsenal des décisions étatiques, leur instrumentalisation objective en tant que systèmes fonctionnels ("industrialo-urbains»), en tant que villes-outils au service de l'État, la remise en question de cette fonction représente pour celles-ci une véritable mutation structurelle qui ne saurait être vécue autrement que sur le mode de la crise (voire de la trahison par une partie de la population locale). Car si crisis veut dire " choix ", il n'est certes pas le fait des premiers concernés dont la marge de manœuvre est extrêmement limitée. C'est le pouvoir central qui se désengage et c'est aux membres de la société locale, pour ainsi dire laissés à eux-mêmes, de se trouver une autre utilité sociale alors même que l'heure est à la mise en concurrence généralisée et à toutes les échelles des différentes parties du monde (Di Méo, 1988 ; Dollfus, 1997 ; Fischer, 1994 ; Penanros, 1995 ; Plihon, 2001). Or, on ne voit pas très bien aujourd'hui ce qui pourrait favoriser Cherbourg, ville presque insignifiante sur la carte internationale des activités et propriétés actuellement valorisées donc valorisantes. Au nom de la maîtrise des coûts économiques, ce sont bien les coûts sociaux et humains de leurs décisions qui sont une fois de plus ignorés ou négligés par ceux qui font et défont les destinées anonymes, d'autant plus facilement que rien ne les en empêche.

\section{BIBLIOGRAPHIE}

Armées d'aujourd'hui, 1999, n²37, février, Délégation à l'Information et à la Communication de la Défense, Paris, éd. ADDIM.

Birnbaum (P.), 1980 (éd. augm.). - Les Sommets de l'État. Essai sur l'élite du pouvoir en France, Paris,

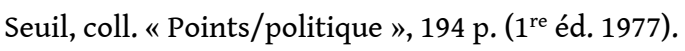

BOLTANSKI (L.), THÉVENOT (L.), 1991. - De la justification. Les économies de la grandeur, Paris, Gallimard, coll. « Nrf Essais », 483 p.

BouRdieu (P.), 1989. - La Noblesse d'État. Grandes écoles et esprit de corps, Paris, Les Éditions de Minuit, coll. « Le Sens commun », 569 p. 
-, 1997. - « De la maison du roi à la raison d'État. Un modèle de la genèse du champ bureaucratique ", Actes de la Recherche en Sciences Sociales, n 118, "Genèse de l'État moderne », p. 55-68.

BROS (Ph.), 1987. - « La question des arsenaux », Défense nationale, novembre, p. 85-102.

BuLÉON (P.), 1986. - Stratégies dans la crise industrielle en Basse-Normandie. Thèse de doctorat, géographie, Université de Caen, 395 p.

CHAPPÉ (F.), 1996. - « La “vocation" maritime de Paimpol ? Création littéraire ou réalité historique ? ", dans Roudaut (F.) (dir.), La Ville maritime. Temps, espaces, représentations, Actes du colloque de Brest des 9-10-11 juillet 1996, Brest, UBO, p. 47-53.

Chesnais (F.), SeRFATi (C.), 1992. - L'Armement en France. Genèse, ampleur et coût d'une industrie, Paris, Nathan, $208 \mathrm{p}$.

COAT (P.), 1982. - Les Arsenaux de la Marine de 1630 à nos jours, Brest/Paris, Les Éditions de la Cité, $199 \mathrm{p}$.

COLLet (A.), 1988. - Les Industries d'armement, Paris, PUF, coll. « Que sais-je ? », 125 p.

DENIS (É.), 1994. - « Cherbourg : un campus en Finisterre. Intégration industrielle ou démocratisation universitaire ? ", dans Robert Hérin (dir.), L'Université, la région, les villes en BasseNormandie, CERA, Université de Caen, p. 175-183.

Di MÉo (G.), 1988. - Les Démocraties industrielles. Crise et mutation de l'espace, Paris, Masson, coll. « Géographie », 244 p.

-, 1991. - L'Homme, la Société, l'Espace, Paris, Anthropos, 319 p.

Dollfus (O.), 1997. - La Mondialisation, Paris, Presses de Sciences Po, coll. « La Bibliothèque du citoyen », $167 \mathrm{p}$.

ÉPIN (Ch.), 1990. - Les Ouvriers des arsenaux de la Marine sous Napoléon. Vivre et survivre en travaillant pour l'État, Montrevillon, L'Herminette, 295 p.

FISCHER (A.), 1994. - Industrie et espace géographique. Introduction à la géographie industrielle, Paris, Masson, $139 \mathrm{p}$.

GARRIGOU (A.), 2001. - Les Élites contre la République. Science Po et l'ENA, Paris, La Découverte, coll. «Cahiers libres », $242 \mathrm{p}$.

GENET (J.-Ph.), 1997. - « La genèse de l'État moderne. Les enjeux d'un programme de recherche », Actes de la Recherche en Sciences Sociales, $n^{\circ} 118$ : «Genèse de l'État moderne », p. 3-18.

GUERMOND (Y.), Muller (C.), 1984. - Le Cotentin d'aujourd'hui, Brionne, Éd Gérard Monfort, 178 p.

HÉBERT (J.-P.), 1995. - « Production d'armement. Mutation du système français », Notes et Études Documentaires, $\mathrm{n}^{\circ}$ 5009-5010, La Documentation française, Paris, $221 \mathrm{p}$. [Cet ouvrage est la version remaniée pour publication de sa thèse d'État: Mutation du système français d'armement : la fin d'une régulation administrée.]

-, 1996. - « 1995 : une année de transition pour l'industrie française d'armement, entre les mutations doctrinales de 1994 et les choix industriels de 1996 ", dans Patrice Bouveret et Belkacem Elomari (dir.), Ventes d'armes de la France. Rapport 1996, Lyon, Observatoire des transferts d'armement, p. 49-58.

LAVAlLEy (J.), LEMONNIER (H.), 1999. - Un Siècle de construction de sous-marins à l'arsenal de Cherbourg. Tome 1 : 1686-1944, Cherbourg, Isoète, 173 p. 
LEBARON (F.), 2000. - La Croyance économique. Les économistes entre science et politique, Paris, Seuil, coll. « Liber », $262 \mathrm{p}$.

LEPELLEY (R.), 1990. - Le Vieil arsenal de Cherbourg (de 1793 à 1814), Cherbourg, Éd. Roger Lepelley sous le patronage de la Société Académique de Cherbourg, 352 p.

LESACHEY (S.), 1992. - « D'un désenclavement géographique à un désengagement gouvernemental dans le Nord-Cotentin », Bulletin du CERA, n 36 : « Emploi : du chômage à la haute technologie », Université de Caen/Basse-Normandie, p. 37-41.

Livre blanc sur la défense, 1994. - préfaces de Édouard Balladur et François Léotard, Paris, La Documentation française, coll. « Les rapports officiels », 263 p.

MANA, Revue de sociologie et d'anthropologie, 1998, $\mathrm{n}^{\circ} 4$ : « Technosciences, risques et vulnérabilité », sous la direction de Frédérick Lemarchand, LASAR, Université de Caen/Basse-Normandie, $266 \mathrm{p}$.

PenanRos (R. de) (dir.), 1995. - « Reconversion des industries d'armement. Crise, adaptation sectorielle et développement régional », Notes et Études Documentaires, n 5020-5021, Paris, La Documentation française, $230 \mathrm{p}$.

Plinon (D.), 2001. - Le Nouveau capitalisme, Paris, Flammarion, coll. « Dominos », 128 p.

PoIsson (S.), 1992. - «Émergence dans le Nord-Cotentin depuis 1985 des services liés à la haute technologie ", Bulletin du CERA, n 36 : « Emploi : du chômage à la haute technologie ", Université de Caen/Basse-Normandie, p. 31-36.

QUELLIEN (J.), 1984. - « Un milieu ouvrier réformiste : syndicalisme et réformisme à Cherbourg à la "Belle Époque" ", Le Mouvement social, n 127, p. 5-88.

RAoulX (B.), 1996. - « Cherbourg et Caen. Deux modèles de relation entre la ville et le port », Norois, $\mathrm{n}^{\circ}$ 169/Études Normandes, $\mathrm{n}^{\circ}$ 1-2 : «La Basse-Normandie », sous la direction de Colette Muller et Michel Siéper, Poitiers/Rouen, p. 93-109.

RIPOLL (F.), 1996. - Cherbourg et le Nord-Cotentin. Des personnels de l'Arsenal aux dynamiques sociospatiales locales, mémoire de maîtrise, CRESO, Université de Caen/Basse-Normandie, 268 p.

RoudAut (F.) (dir.), 1996. - La Ville maritime. Temps, espaces, représentations, Actes du colloque de Brest des 9-10-11 juillet 1996, Brest, UBO, 359 p.

SÉCHET (R.), 1992. - « Ports militaires : les arsenaux passent le relais aux universités », Annales de la recherche urbaine, $\mathrm{n}^{\circ}$ 55-56, p. 88-95.

SIEGFRIED (A.), 1995. - Tableau politique de la France de l'Ouest sous la Troisième République, Paris, Imprimerie Nationale, 636 p. (1 ${ }^{\text {re }}$ éd. 1917).

TAillemite (É.), 1991. - « Cherbourg. Des projets de Vauban aux sous-marins nucléaires », Cols Bleus, $n^{\circ} 2139$, p. 4-9.

TOLLEMER (G.), 1997. - Cherbourg ville d'État. Forces et faiblesses du second pôle bas-normand, mémoire de maîtrise, GÉOSYSCOM, Université de Caen/Basse-Normandie, 109 p.

VIGARIÉ (A.), 1988. - « Lorient, Brest, Cherbourg : un conflit permanent entre commerce et puissance navale? », Hommes et terres du Nord, numéro hors série : "Campagnes et littoraux d'Europe. Mélanges offerts à Pierre Flatrès ", Société de géographie de Lille, p. 114-122. 


\section{NOTES}

1. Titre d'un article d'un contrôleur général des armées (Bros, 1987). Le terme arsenal est le nom originel des manufactures d'armes puis des chantiers de construction navale militaire. Il est encore utilisé communément pour désigner ces derniers.

2. Créé «pour remplacer Brouage envasé » (Collet, 1988, p. 10), il fermera ses portes en 1926 (Coat, 1982).

3. « Où la valeur ajoutée est relativement plus faible que dans les autres secteurs » (Hébert, 1995, p. 103).

4. La recherche de maîtrise, d'où est issu l'essentiel de ces paragraphes, tente de mesurer la part prise par les personnels de l'arsenal de Cherbourg dans l'histoire des dynamiques socio-spatiales locales.

5. Selon la période, au $\mathrm{xx}^{\mathrm{e}}$ siècle surtout, ce sont les conditions de travail, la formation et la mobilité internes, la sécurité de l'emploi, le salaire ou la retraite, qui, seuls ou combinés, ont pu représenter un avantage relatif, parfois objectif, parfois mythifié, par rapports aux autres catégories locales. Quoi qu'il en soit, ces « acquis sociaux » ont été conquis par des luttes parfois dures, pour changer des conditions de vie déplorables (Coat, 1980; Épin, 1990 ; Lavalley, Lemonnier, 1999) et sont aujourd'hui contournés et remis en question.

6. Établissement le plus important, la Cit-Alcatel a réduit le nombre de ses salarié(e)s de 2000 à 1 450 entre 1977 et 1984, date des premiers licenciements. En 1990, ils/elles n'étaient plus que 665. L'établissement a fermé ses portes en 1996. L'UIE a fermé dès 1985.

7. Construit par le CEA (Commissariat à l'Énergie Atomique) en 1962 et géré par la COGEMA (Compagnie Générale des Matières nucléaires) depuis 1976.

8. Voir par exemple les articles de Laurent Boceno et Guillaume Grandazzi dans Mana, 1998.

9. Pour reprendre les exemples de La Ville maritime. Temps, espaces, représentations, 1996.

10. Pour une présentation de ce « concept-outil », développé surtout par Guy Di Méo (1991).

11. À Cherbourg, selon Éric Denis, «l'université n'est pas venue relayer une activité navale déclinante, offrant des ouvertures, mais elle s'est affirmée comme le levier qui allait permettre d'adapter la main d'œuvre locale et exogène aux exigences d'un appareil industriel parmi les plus sophistiqués d'Europe occidentale. Le développement des formations supérieures ne s'inscrit donc pas dans une logique de reconversion. [...] L'université ne modifie donc pas les termes de la dépendance à l'égard des grands donneurs d'ordre » (Denis, 1994, p. 176-177).

12. Il aura fallu plus de 40 ans pour que la mise en 2 x 2 voies de la RN 13 entre Cherbourg et Caen, réclamée depuis les années 1960, soit enfin terminée, et ceci, « malgré un coup de fouet aux travaux dû aux festivités prévues lors de la commémoration du cinquantenaire du débarquement du 6 juin 1944 » (Tollemer, 1997, p. 96). Et maintenant que cela est fait, la nouvelle « autoroute des estuaires » (A84) produit un nouvel enclavement relatif : en passant au sud de la Manche sans rejoindre la RN 13, elle favorise Caen au détriment de Cherbourg.

13. 5,5 hectares ont été gagnés sur la mer pour pouvoir implanter l'atelier Coque (fabrication, formage et soudage des éléments), le chantier Laubeuf (préfabrication lourde et assemblage des éléments) et le DME (Dispositif de Mise à l'Eau à la verticale opéré après transfert du sous-marin à l'horizontal depuis le chantier à l'aide de marcheurs hydromécaniques).

14. Le nombre de SNLE-NG est ramené de six à quatre et leur programme est étalé. Celui des SNA (sous-marins nucléaires d'attaque) est ramené de huit à six alors que le septième est en cours de construction.

15. Ceci, qui est vrai en période stable c'est-à-dire en période de rapports de force stabilisés, l'est encore plus en période d'instabilité voire de crise où l'indétermination objective, l'incertitude subjective et l'imprévisibilité des actions des uns et des autres (et des uns par les autres) sont maximales. C'est précisément le cas pendant au moins les cinq décennies qui voient naître le 
Cherbourg militaro-industriel, alors que se succèdent voire se conjuguent crises économiques, conflits sociaux, révolutions politiques, coups d'État, guerres.

16. L'évaluation inclut le repérage des données pertinentes, leur classification positive (bien, avantage, gain) ou négative (mal, inconvénient, coût), leur hiérarchisation, la détermination des priorités, de (ce) qui ne peut pas et ne doit pas être sacrifié, et inversement, de (ce) qui peut et doit l'être...

17. Napoléon Bonaparte n'était-il pas «résolu à renouveler à Cherbourg les merveilles de l'Égypte " (si l'on en croit cette phrase citée dans tous les livres d'histoire locale et inscrite sur le socle de la statue équestre qui lui est dédiée à Cherbourg)?

18. Comme nombre d'ingénieurs des autres secteurs, publics ou privés, les IA ont des fonctions qui vont bien au-delà de la seule conception technique. Ils occupent les plus hauts postes décisionnels de la DGA, de ses différentes directions (dont la DCN) et des établissements industriels (dont les arsenaux).

19. Avec son opération "ville morte " (commerces fermés, débrayages et participation aux mobilisations d'autres salariés et des familles), le conflit de 1992 est très clairement construit comme «conflit de bassin d'emploi ». Il n'y avait d'ailleurs pas eu de conflit social important depuis 1979 (Cf. Buléon, 1986).

20. Jacques Chirac, Président de la République, déclaration faite le 22 février 1996, site internet du ministère de la Défense.

21. DGA, site Internet du Ministère de la Défense.

22. Selon Jean-Philippe Genet, la guerre est un "puissant agent de cohésion de la société politique, qui joue un véritable rôle moteur dans l'évolution de l'État moderne. La guerre est le moteur de l'État moderne qui, avant tout, est un État de guerre » (Genet, 1997, p. 4).

23. Ce travail prolonge une thèse de doctorat soutenue en 1996 : Les Économistes français entre science et politique. Contribution à une sociologie de la culture économique). Sur les «grandes écoles » et leurs évolutions, voir aussi Bourdieu, 1989 ; Garrigou, 2001.

24. Il n'est pas anodin de retrouver la critique de l'étatique (empirisme, dysfonctionnements, rigidité, handicap...) et l'apologie de l'économique (logique, remède, émancipation, avantage...) dans un article déjà ancien d'un contrôleur général des armées (Bros, 1987). Ni de lire qu'une grande part des membres du comité exécutif de DCN sont passés par le secteur privé et/ou présentent une compétence commerciale (site Internet de «l'entreprise » DCN).

25. Dans La Ville maritime (1996), alors que d'autres participants du colloque en usent tel quel, l'historien François Chappé met rapidement et radicalement en question «ce langage aussi téléologique que théologique qui parle de vocation » (p. 51) pour demander plus loin «toutes les précautions de vérification dont les moindres ne sont pas celles qui portent sur le langage.» (p. 53). Paradoxalement, l'analogie religieuse est ici d'autant plus intéressante que les verdicts des plus hautes autorités sociales - faits au nom du droit divin, de la raison d'État ou aujourd'hui de la rationalité économique - sont souvent présentés comme des jugements derniers, et ont de véritables effets de destin.

\section{RÉSUMÉS}

Qui veut rendre compte de l'industrie française d'armement - des caractéristiques de son fonctionnement, des causes et conséquences des importantes restructurations en cours - ne peut se limiter à des considérations d'ordre géopolitique, économique ou technologique, ni se focaliser 
sur l'échelle nationale ou internationale. À partir du cas concret de la «ville-arsenal » de Cherbourg, de sa naissance à ses déboires actuels, ce texte vise à dégager quelques lignes de force en inscrivant la réflexion dans une histoire et une géographie sociales de la construction navale militaire... et donc de l'État français. Quelques pistes factuelles et méthodologiques sont ainsi lancées pour mettre en question la « fatalité » des dynamiques socio-spatiales passées et à venir. Que penser en effet de la «nécessité » des décisions prises par les dirigeants? Que penser de celles qu'ils ne prennent pas? Quelle probabilité pour les Cherbourgeois de construire un développement économique alternatif et endogène?

No study on French arms industry - on the characteristics of its working, on the causes and consequences of its serious current restructuring - should neither be limited to geopolitical, economical or technological considerations, nor focus on international or national scales. Through the concrete case of the "ville-arsenal" of Cherbourg, from its birth to its present difficulties, this paper tries to point up some of the main features which are included in the frame of a social history and geography of military shipbuilding... and then of the French state. Some historical facts and methodological tools are proposed to question the "fatality" of the past and future socio-spatial dynamics. What should we think about the "necessity" of the government's decisions? And what about the decisions which are not taken? What is the probability to see the inhabitants of Cherbourg build an alternative and endogenous economical development?

\section{INDEX}

Index géographique : Cherbourg, France

Keywords : globalization, military field, naval dockyards, privatization, socio-spatial configuration, state

Mots-clés : champ militaire, industrie française d'armement, mondialisation, configuration socio-spatiale, État, restructuration

\section{AUTEUR}

\section{FABRICE RIPOLL}

Université de Caen Basse-Normandie, CRESO (Centre de Recherche sur les Espaces et les Sociétés)

- UMR ESO, ripoll@mrsh.unicaen.fr 